\title{
La Cohésion Sociale En Côte d'Ivoire: Analyse De La Situation Et Facteurs Selon Les Ivoiriens
}

\author{
Sali Lokotianwa Yeo Kone \\ Docteur en Psychologie Sociale \\ Maître-Assistant au département de Psychologie \\ Université Félix Houphouët Boigny, Côte d'Ivoire
}

Doi: 10.19044/esj.2018.v14n8p267 URL:http://dx.doi.org/10.19044/esj.2018.v14n8p267

\begin{abstract}
This study proposes to examine the situation of social cohesion in Côte d'Ivoire. To do this a survey is conducted on a sample of 1536 Ivoirians in the four major regions of Côte d'Ivoire in both rural and urban areas. This sample is composed of 60 ethnic groups grouped into four (04) groups. The size of the sample obtained by the quota method (region, sex, agglomeration, age (18 years and over) socio-professional situation) is calculated taking into account the data of the last General Census of Population and Housing (RGPH., 2014). The results reveal that the Ivorian aware of his role also relies on public authorities to reduce disparities and polarizations. This will give people the impression that they are involved in a common enterprise, that they face the same challenges and are part of the same community.
\end{abstract}

Keywords: Social cohesion, categorical differentiation

\section{Resume}

Cette étude se propose d'examiner la situation de la cohésion sociale en Côte d'Ivoire. Pour ce faire une enquête est menée sur un échantillon de 1536 ivoiriens dans les quatre grandes régions de la Côte d'Ivoire aussi bien dans le milieu rural qu'Urbain. Cet échantillon est composé de 60 ethnies regroupées en quatre (04) groupes. La taille de l'échantillon obtenu par la méthode des quotas (région, sexe, agglomération, âge (18 an et plus) situation socioprofessionnelle) est calculée en tenant compte des données du dernier Recensement Générale de la Population et de l'Habitat (RGPH, 2014).

Les résultats révèlent que l'ivoirien conscient de son rôle compte aussi sur les pouvoirs publics pour réduire les disparités et les polarisations. Ce qui permettra aux ivoiriens d'avoir l'impression qu'ils participent à une entreprise commune, qu'ils ont les mêmes défis à relever et qu'ils font partie de la même collectivité. 
Mots-clés : Cohésion sociale, différenciation catégorielle, conflit, représentation

\section{Introduction}

Après les nombreuses crises qu'a connues la Côte d'Ivoire (coup d'état en 1999, rébellion en 2002, et crise poste électorale en 2010) et l'instauration de la troisième république, un constat demeure. La réconciliation qui conduira la cohésion rencontre toujours des obstacles. Des actions sont menées pour réconcilier les ivoiriens, notamment celles des gouvernants. En effet, par le biais du ministère en charge de la cohésion sociale, des campagnes de sensibilisation dans les médias (télévision, radio, réseaux sociaux, etc.) sont faites dans les différentes régions pour inciter les populations à la cohésion sociale. Cependant, malgré toutes ces actions, l'on constate que des situations les plus anodines débouchent fréquemment sur des affrontements entre différentes communautés dans plusieurs localités du pays ${ }^{35}$. Ces mouvements de colère engendrent régulièrement la destruction de biens publics et privés. Dans une telle ambiance de tensions sociales, nous sommes amenés à nous poser certaines questions. Quel est l'état des lieux en ce qui concerne la cohésion sociale en Côte d'Ivoire ? Quels sont les facteurs de la cohésion sociale selon les ivoiriens ? Comment parvenir à cette cohésion sociale et à la réconciliation nationale en Côte d'Ivoire selon les concernés ?

Pour répondre à ces questions, nous posons le problème dans la problématique, ensuite nous exposerons la méthodologie de recueil des données et enfin nous présenterons les résultats de l'enquête et la discussion de ceux-ci.

\section{Problématique}

La cohésion sociale est une notion d'actualité. Portée par les institutions internationales et européennes, elle s'est imposée en Afrique notamment en Côte d'Ivoire. Face à l'accroissement des inégalités sociales et la complexité de la gestion des diversités culturelles, les difficultés qui se concentrent dans certains territoires ou auprès de certaines catégories de population, comment analyser et lire les problématiques sociales émergentes ?

Poser la question de la cohésion sociale dans ces conditions, c'est poser celle de la nature et de l'intensité des relations existant au sein d'une société. La cohésion sociale, selon Durkheim (1893), est un processus permanent qui consiste à établir des valeurs communes et des objectifs communs et à offrir

\footnotetext{
${ }^{35}$ Affrontement le lundi 2 octobre 2017 entre les Baoulé et Wè à l'ouest de la Côte d'Ivoire faisant 2 morts 8 blessés dont 3 graves et des dizaines de déplacés (télévision ivoirienne première chaine journal de 20 heure).
} 
des chances égales se fondant sur un idéal de confiance, d'espoir et de réciprocité parmi tous.

La cohésion sociale ne saurait vouloir dire simplement une société homogène sans diversité. Ceci indique une euphémisation de la question sociale, qui devient moins centrale, et une nouvelle orientation de la réflexion sur le lien social autour de la notion de confiance: confiance entre les individus et les institutions, et confiance entre les individus.

A l'aube de l'indépendance, à l'instar des autres pays sous domination coloniale, la Côte d'Ivoire baignait dans l'euphorie de la liberté retrouvée et l'espoir d'un développement rapide et harmonieux. Certes, les deux premières décennies ont rendu possible ce que d'aucuns ont appelé le «miracle économique » Ivoirien. Des infrastructures modernes (routes, écoles, dispensaires hôpitaux, industries agro-économiques etc.) sont réalisées. Le pouvoir d'achat de l'Ivoirien s'est significativement amélioré grâce à une croissance économique relativement élevée.

Mais à la fin des années 1980, la situation économique va se dégrader avec de graves conséquences sociales. Les mécontentements, les frustrations et l'injustice ressentie par les populations ivoiriennes se sont accentués. Ces ressentiments se manifesteront davantage pour déboucher sur des revendications sociopolitiques. Cette situation va créer une méfiance réciproque, des tensions sociales, la fracture entre différentes communautés ethniques et donc la catégorisation des uns et des autres.

Le processus de catégorisation sociale va engendrer et renforcer le processus de différenciation catégorielle entre ivoiriens. Cette différenciation sociale, en tant que processus cognitif nous semble un enjeu réel dans le processus des relations intergroupes, dès lors qu'elle soulève ici, la question de l'identité sociale et individuelle de l'ivoirien.

Le terme catégorie se rapporte au concept de catégorisation qui se définit selon Tajfel (1972) comme le processus psychologique qui tend à ordonner l'environnement en termes de catégories, groupes de personnes, d'objets, d'événements, en tant qu'ils sont soit similaires, soit équivalents les uns aux autres pour l'action, les intentions ou les attitudes d'un individu.

Pour Deschamps (1977), la catégorisation sociale est le phénomène qui rend compte de la division entre le « Nous » et le « Eux », entre le « In-group » et le « Out-group ». Cette distinction se retrouve à travers des groupements de toutes sortes d'après Bazoumana (2002), qu'ils soient sociaux, économiques, nationaux, raciaux, ethniques, d'âge, de sexe, etc.

Selon Marchand (1970), le processus de catégorisation implique une simplification de la réalité qui se fait grâce à deux mouvements complémentaires, accentuation des ressemblances entre les éléments d'une même catégorie et celle des différences entre les catégories. 
Le contenu des catégories et l'organisation catégorielle ne sont pas stables mais sont le produit d'une perception. Par exemple, une voiture peut être classée dans la catégorie "moyen de transport" si l'on considère l'objectif d'un sujet qui est de chercher à se déplacer; ou dans d'autres circonstances, dans la rubrique siège si l'objectif est de «chercher à s'asseoir ». Ceci induit que la valeur de la catégorie déteint sur les objets qu'elle contient (Bruner et Goodman, 1947 ; Bruner, 1957).

Le processus de catégorisation sociale joue un rôle primordial. Tajfel définit la catégorisation sociale comme un système d'orientation qui crée et définit la place particulière d'un individu dans la société (1972). Il permet à l'individu de reconnaître la manière dont la réalité sociale est divisée en catégories, et surtout, de savoir quelles sont les catégories auxquelles il appartient et quelles sont celles auxquelles il n'appartient pas.

C'est d'ailleurs pour cela que Bazoumana (1985) affirme que la simple catégorisation est une condition suffisante pour entraîner une forme de comportement discriminatoire. Dans un tel contexte on comprend mieux que les conflits $^{36}$ deviennent de plus en plus exacerbés et ouverts entre les populations.

Le mot « conflit » vient du latin « conflictus » qui signifie : heurt, choc, lutte, attaque.

Il s'applique, à l'origine, à une situation de lutte armée, de combat entre deux ou plusieurs personnes, organisations ou puissances, qui se disputent un pouvoir.

Par extension, le terme de conflit s'applique aujourd'hui à toute opposition survenant entre des parties en désaccord, l'une souhaitant imposer ses positions, à l'encontre des attentes ou des intérêts de l'autre partie.

$\mathrm{Au}$ sens légal, un conflit est un contentieux sur un ou des points de droit. Selon Picard et Marc (2008), le conflit est l'affrontement de deux ou plusieurs volontés individuelles ou collectives qui manifestent les unes à l'égard des autres une intention hostile et une volonté d'agression, à cause d'un droit à recouvrer ou à maintenir.

Il peut être de plusieurs types : interpersonnel ; intra personnel ; intergroupe ; intragroupe. Il peut avoir plusieurs sources : les intérêts ; les conflits de pouvoir; les conflits identitaires; les conflits territoriaux; les conflits de relation ; les conflits cognitifs ; les conflits affectifs ; les conflits culturels. Lewin (1997) affirme que le champ influence durablement le comportement des individus.

\footnotetext{
${ }^{36}$ Conflit foncier dans l'ouest de la Côte d'Ivoire à Guiglo précisément à Dramankro un petit village de la forêt classée de Goin- Débé le mardi 03 et jeudi 04 Octobre 2017 (2 morts et 8 blessés).

Affrontement agriculteurs et éleveurs peules à Bouna au nord-est du pays les 24 et 25 mars 2017 (20 morts 30 blessées et environ 2000 déplacés).
} 
Ce champ n'est pas composé de l'environnement physique et social seulement, il comprend une composante cognitive qui détermine une sorte d'environnement psychologique (perception). Aussi, les difficultés que les uns et les autres rencontrent (problème de terres cultivables, croissance de la pauvreté, etc.) ; les frustrations de la vie pousse les ivoiriens à se regarder en chien de faïence. Alors, quels sont les facteurs de la cohésion sociale et comment y parvenir en Côte d'Ivoire ?

La réponse à cette question nous permettra d'analyser l'état de la question actuellement en Côte d'Ivoire; de définir la représentation /perception de la cohésion sociale en Côte d'Ivoire; de définir ces indicateurs et de définir les conditions pour atteindre la cohésion selon les ivoiriens.

\section{Méthodologie}

\section{Participants}

Cette étude porte sur la population ivoirienne dans son ensemble. Cette population, estimée à environ vingt un (21) million d'habitants selon l'Institut National de la Statistique (INS, 2014), est composée de 60 ethnies regroupées en quatre (04) groupes. L'échantillon est composé de 1536 personnes sélectionnées selon la méthode des quotas. Ces quotas (région, sexe, agglomération, âge (18 an et plus) situation socioprofessionnelle) ont été calculés en tenant compte des données du dernier Recensement Générale de la Population et de l'Habitat (RGPH, 2014).

\section{Matériel}

L'enquête a été réalisée pendant douze mois avec un questionnaire aussi bien dans les milieux ruraux qu'urbain. Un guide d'entretien est utilisé subsidiairement pour approfondir certaines informations du questionnaire et aider dans l'interprétation des résultats. La construction de ces instruments s'est faite en prenant en compte sept axes. L'un définit la représentation de la cohésion sociale chez les sujets étudiés et les conditions qui sont indispensable à la cohésion, l'autre fait une analyse de la situation actuelle à travers les acteurs, les facteurs qui doivent être conjugués pour l'atteinte de la cohésion sociale.

Après l'enquête, nous obtenons les résultats qui suivent.

\section{Résultats}

Les résultats sont présentés en 7 axes. Chaque axe est représenté par les réponses des sujets ainsi que le pourcentage dans lequel ces réponses sont données. 


\section{Présentation}

Première question : Quels sont les mots qui vous viennent à l'esprit lorsqu'on vous parle de cohésion sociale ? Question ouverte réponses spontanées.

$\begin{array}{cc}\text { Tableau 1 : réponses } & \\ \text { Evocations } & \text { Pourcentages } \\ \text { Les aspects relationnels } & 56 \% \\ \text { Les aspects économique et social } & 25 \% \\ \text { La mise à distance de l'idée de cohésion sociale } & 19 \%\end{array}$

Les aspects relationnels avec $56 \%$ sont les suivant.

- Vivre en bonne entente sans conflit

- $\quad$ Solidarité entraide

- $\quad$ Respect des autres

- $\quad$ Etre tolérant

- $\quad$ Unité de la population

- $\quad$ Lien social (les gens se parlent)

Les aspects économiques représentent $25 \%$

- $\quad$ Transparence dans la gestion des affaires publiques (étatiques)

- $\quad$ Egalité, lutte contre les inégalités

- $\quad$ Partage richesse

- $\quad$ Condition de vie descente a tous

La mise à distance de l'idée de cohésion sociale 19\%.

- Utopie inatteignable

- $\quad$ Leurre politique

Le premier constat c'est que l'expression "cohésion sociale" est très évocatrice puisque $92 \%$ des sujets en proposent spontanément une définition. Elle renvoie selon les sujets interrogés aux relations harmonieuses entre membres de la société (56\% des participants). Ensuite, la dimension économique de la cohésion sociale est abordée en deuxième position par $25 \%$ des sujets. Les notions d'égalité entre les citoyens dans le partage des richesses ou d'équité des conditions de vie (logement, travail, éducation, soin etc.). L'expression suscite également une mise à distance de la part de $19 \%$ des sujets. Certain parle de leurres et d'autre exprime une réserve.

Deuxième question : Pour vous quelles sont les conditions les plus indispensables à la cohésion sociale ? (ouverte) 
Tableau 2 : réponses

\begin{tabular}{|c|c|}
\hline $\begin{array}{c}\text { Evocations } \\
\text { autres }\end{array}$ & Pourcentages \\
\hline $\begin{array}{c}\text { les citoyens doivent s'accepter et se respecter les uns les } \\
\text { Les citoyens doivent partager les mêmes valeurs }\end{array}$ & $31 \%$ \\
\hline les citoyens doivent être solidaires entre eux & $18 \%$ \\
\hline les citoyens doivent avoir un projet commun d'avenir & $11 \%$ \\
\hline La justice doit être impartiale & $10 \%$ \\
\hline $\begin{array}{c}\text { les lois doivent s'appliquer de la même façon à tous les } \\
\text { citoyens }\end{array}$ & $10 \%$ \\
\hline
\end{tabular}

- les citoyens doivent s'accepter et se respecter les uns les autres (31\%).

-Les citoyens doivent partager les mêmes valeurs $(20 \%)$.

-les citoyens doivent être solidaires entre eux (18\%).

- les citoyens doivent avoir un projet commun d'avenir (11\%).

- La justice doit être impartiale (10\%).

- les lois doivent s'appliquer de la même façon à tous les citoyens (10\%).

Le respect mutuel paraît comme la condition la plus importante avec $31 \%$ des suffrages. "Les citoyens doivent se respecter les uns les autres » introduit une notion de réciprocité pour bâtir la cohésion sociale, il faut accepter de reconnaître à chacun sa valeur. En un mot, j'attends pour moimême autant de considération que j'en dois à autrui Hoibian (2011).

La notion d'unité intervient beaucoup. Les citoyens doivent avoir des valeurs qui les unissent.

Elle peut signifier l'unité des valeurs, des normes, un projet commun d'avenir en vue d'objectifs partagés. La lutte contre les inégalités interviennent aussi avec "la justice doit être impartiale". La cohésion intègre le vivre ensemble, la citoyenneté, le bien-être et la solidarité.

Troisième question: Selon vous, aujourd'hui en Côte d'Ivoire qu'est ce qui contribue le plus au renforcement de la cohésion sociale ? (ouverte)

Tableau 3 : réponses

\begin{tabular}{|c|c|}
\hline Evocations & Pourcentages \\
\hline $\begin{array}{c}\text { - La volonté de chaque ivoirien de vivre ensemble avec ses } \\
\text { frères (les ivoiriens sont un peuple de paix) }\end{array}$ & $43 \%$ \\
\hline $\begin{array}{c}\text { - La libération des prisonniers politiques/ prisonniers de la } \\
\text { crise post-électorale }\end{array}$ & $25 \%$ \\
\hline L'indemnisation des victimes des différentes crises & $16 \%$ \\
\hline Impartialité de la justice & $13 \%$ \\
\hline $\begin{array}{c}\text { Education scolaire à la paix, la tolérance et l'acceptation des } \\
\text { autres }\end{array}$ & $03 \%$ \\
\hline
\end{tabular}

- La volonté de chaque ivoirien de vivre ensemble avec ses frères (les ivoiriens sont un peuple de paix) (43\%) 
- La libération des prisonniers politiques/ prisonniers de la crise post-électorale (25\%)

- L'indemnisation des victimes des différentes crises $(16 \%)$

-Impartialité de la justice (13\%)

- Education scolaire à la paix, la tolérance et l'acceptation des autres (3\%)

L'individu est considéré comme le moteur de la cohésion sociale "la volonté de chaque ivoirien de vivre ensemble avec ses frères" vient loin devant avec $43 \%$. L'allusion à l'indemnisation des victimes, l'éducation scolaire à la paix, la libération des prisonniers de la crise post-électorale, fait ressortir le rôle aussi important que jouent les gouvernants dans l'atteinte de la cohésion sociale. Ce fait se comprend bien pour la Côte d'Ivoire qui vient de vivre une crise sans précèdent.

Quatrième question : La cohésion sociale est-elle une réalité en Côte d'Ivoire ? (Question avec proposition de modalités de réponses)
Pas du tout forte
Tableau 4 : Modalité de réponses choisies
$35 \%$ des sujets interrogés
Pas très forte
Assez forte
$25 \%$ des sujets enquêtés
$40 \%$ des sujets

L'individualisme est assez présent. Les indicateurs intégrés dans l'enquête révèlent une réalité nuancée. $40 \%$ des enquêtés suggèrent qu'il est possible de faire confiance aux autres alors que $35 \%$ considèrent l'inverse. Cette question révèlent un clivage entre catégorie socioprofessionnelle aisée et diplômée penchant plutôt du côté de la confiance tandis que les groupes modestes (travailleurs du secteur informel, handicapées, chômeurs, les personnes vivant dans le milieu rural) sont méfiants.

En plus, nous avons constaté que la réponse des personnes variait selon leur appartenance politique. Aussi, les personnes proches du pouvoir actuelle estiment-elles $80 \%$ que la cohésion existe et elle est forte selon eux. Par contre, lorsqu'il s'agit de ceux de l'opposition, ils affirment le contraire.

Cinquième question : Auxquels de ces lieux avez-vous le sentiment d'appartenir avant tout ? (question avec proposition de réponses)

Tableau 5 : Modalités de réponses choisies

A votre région $27 \% \quad$ A votre village/département 34\% A la Côte d'Ivoire 39\%

- A votre village /ville / commune / département $\quad 34 \%$

- A votre région $27 \%$

-A la Côte 'Ivoire39\%

La force du lien entre les membres de la société se mesure aussi à travers le sentiment d'appartenance au pays. Plus ce lien est fort, plus forte est la cohésion. Le sentiment d'appartenance au département $34 \%$ (village, ville d'origine) est sensiblement le même que celui du pays 39\%, l'appartenance la région vient derrière avec $27 \%$ des suffrages. 


\section{Sixième question : Selon vous, que doit faire les pouvoirs publics pour renforcer la cohésion?}

Tableau 6 : réponses

Evocations

- Assurer à tous la possibilité d'occuper un emploi

(éviter le tribalisme pour les postes administratifs

permettre à tous de bien se loger

aider les jeunes à démarrer dans la vie

garantir à tous la possibilité de se soigner

travailler ensemble avec l'opposition

Pourcentages

$75 \%$

$65 \%$

$60 \%$

$47 \%$

$35 \%$

$30 \%$

punir sévèrement les auteurs de détournements de deniers publics

avoir une politique culturelle dynamique qui permet des moments de partage entre les citoyens

soutenir les populations vulnérables et leurs familles

faire revenir les refugies ivoiriens
$23 \%$

$17 \%$

$09 \%$

- Assurer à tous la possibilité d'occuper un emploi (éviter le tribalisme pour les postes administratifs)

-permettre à tous de bien se loger

-aider les jeunes à démarrer dans la vie

-garantir à tous la possibilité de se soigner

-travailler ensemble avec l'opposition

-punir sévèrement les auteurs de détournements de deniers publics

-avoir une politique culturelle dynamique qui permet des moments de partage entre les citoyens

-soutenir les populations vulnérables et leurs familles

-faire revenir les refugies ivoiriens

Septième question: Quels sont les acteurs les mieux placés pour régler le problème de la cohésion ? (question ouverte)

Tableau 7 : réponses

Evocations

Les pouvoirs publics (gouvernement,

services sociaux et publics spécialisés,

départements ou régions)

Les habitants eux-mêmes

La solidarité
Pourcentage

$75 \%$

$19 \%$

$06 \%$

Les pouvoirs publics sont considérés comme les plus à même de régler le problème de cohésion sociale. Lorsqu'on demande à la population de designer les acteurs les mieux placés pour améliorer la cohésion sociale, le gouvernement arrive en tête des réponses

(57\%) des suffrages, les services sociaux et services publics spécialisés $9 \%$, les départements ou régions $9 \%$. Au total, $75 \%$ de la population attendent 
une action des pouvoirs publics, contre 19\% qui s'en remettent aux habitants eux-mêmes, ou 6\% aux solidarités familiales.

Les ivoiriens demandent principalement à la puissance publique la garantie d'accès pour tous à l'emploi (51\%) et au logement $(30 \%)$, des domaines dans lesquels nos concitoyens rencontrent beaucoup difficultés.

\section{Analyse et discussion des résultats}

Le premier constat qui s'impose à la lecture des résultats est la richesse des évocations liées au concept. $92 \%$ de la population interrogées en proposent une définition. Les évocations se rapportent à trois dimensions.

Le principal registre d'évocation a trait aux relations entre membres de la société ivoirienne $56 \%$. Ces derniers définissent la cohésion sociale comme la capacité de la population à "vivre ensemble" en bonne entente sans "conflit" La notion d'unité apparaît ici comme fondamentale. La cohésion se définit comme la propriété d'un ensemble unité dont toutes les parties sont intimement unies. Celle-ci peut tout à la fois signifier une unité des valeurs, des normes, un projet commun. Cela se réfère à la notion de capital social défini par Robert (1995) comme « un ensemble de relations, de valeurs et de normes communes e de confiance qui permet une action collective en vue d'objectifs partagés » (p. 664).

La solidarité, l'entraide permet de se serrer les coudes. Cette vision rejoint celle de Mauss(2007) pour qui le don et le contre-don entretiennent la cohésion du groupe. Cette entraide doit se faire dans le respect de l'autre, dans la tolérance et l'unité.

La cohésion sociale revêt une dimension économique pour $25 \%$ des enquêtés. Il s'agit du partage équitable des richesses, de la réduction des inégalités pour aboutir à des conditions acceptables de vie.

Cependant, le terme ne fait pas consensus ; 19\% des sujets expriment une forte réserve. Certains jugent l'idée irréaliste 10\% et d'autres $9 \%$ n'hésitent pas à la qualifier de "leurre politique" ou de "baratin". Enfin, 8\% des enquêtés ne savent pas quelle signification attribuer au concept.

L'expression est d'autant plus porteuse de sens que l'on est mieux placé dans l'échelle sociale. Les diplômés, personnes avec des revenus élevés, cadres et professions intellectuelles supérieurs se montrent les plus prolixes.

Lorsqu'on compare les effets du sexe, de l'âge, du diplôme, des revenus de la profession et de la taille de l'agglomération de résidence, le niveau d'étude se révèle le critère le plus explicatif des réponses. Un diplômé supérieur à trois fois plus chance d'avoir une idée sur le concept qu'une personne ayant pour diplôme le BEPC.

Les hommes sont les plus nombreux à proposer une définition du concept que les femmes. Cet écart est confirmé en contrôlant les effets du diplôme et de la profession. Cela se comprend mieux dans le contexte ivoirien 
ou les femmes sont peu nombreuses à occuper les postes supérieurs dans les entreprises mais aussi par le faite qu'elles ne sont pas suffisamment scolarisées.

La notion est sujette à des variations notables selon la politisation des individus. Les sujets proches de la coalition au pouvoir la trouvent assez bonne. Par contre, ceux qui sont proches de l'opposition la juge inexistence ou la qualifie de baratin.

Les classes les plus défavorisées (soit 60\% des personnes à revenu économique faible) sont les plus nombreuses à évoquer les notions de solidarité d'entraide, de lutte contre les inégalités et de partage des richesses. Selon ces dernières, les inégalités vont en se creusant. Elles considèrent que les personnes les plus favorisées sont de plus en plus favorisées et les défavorisées de plus en plus défavorisées. L'iniquité sociale trouve un écho dans nombreux domaine. Par exemple, elles estiment à $86 \%$ qu'il existe une médecine une école pour les riches et une autre pour les pauvres.

Si l'identification politique influe sur la connaissance du concept, elle génère en revanche assez peu de divergence de contenu. La dimension du lien entre les individus prédomine. Comment établir des relations harmonieuses entre les individus?

Le rôle des pouvoirs publics s'avère primordiale dans la mesure où ils doivent assurer à tous la possibilité d'occuper un emploi et la protection en facilitant l'accès au logement, et en garantir la protection sociale des individus. Les gouvernants doivent être ouverts en faisant participer tous les ivoiriens au développement de leur pays.

La cohésion sociale s'obtient par la confiance entre les individus et entre les individus et les institutions. Le respect envers les institutions, d'après Paugam (2007), doit prendre appui sur la volonté manifeste de l'État de réduire les inégalités sociales et instaurer la confiance horizontale. Dacheux et Goujon (2010) définissent la confiance horizontale comme celle qui prend appui sur la communauté en mettant l'acens sur la promotion du local, du quartier, du territoire de l'agglomération en associant les habitants à l'élaboration d'un projet commun.

Hors, les agressions multiples contre les symboles de l'Etat que l'on observe (attaque de commissariats, saccage de brigade de gendarmerie etc.) dès qu'il y a un mécontentement montre un ras-le-bol de la population vis-àvis des autorités et le manque de confiance vis-à-vis des institutions.75\% des personnes interrogées estiment que les pouvoirs publics ne jouent pas leur rôle.

\section{Conclusion}

Les résultats auxquels nous sommes parvenus révèlent que les ivoiriens sont conscients du fait que la cohésion sociale relève certes des efforts individuels, mais s'appuie sur les institutions. $25 \%$ des enquêtés 
estiment que la cohésion n'est pas très forte et 35\% qu'elle est pas du tout forte. Cela révèle un clivage dans les opinions.

Pour eux, les gouvernants doivent dépasser le périmètre traditionnel de l'action social qui intervient traditionnellement en réparation des ruptures pour s'intéresser aux modes de création des liens sociaux, intégrant "le vivreensemble" et la citoyenneté. A ce titre, la participation à la vie sociale, que ce soit à travers l'engagement politique, associatif, ou syndical, peut en constituer un marqueur. De façon générale, les gens doivent avoir l'impression qu'ils participent à une entreprise commune, qu'ils ont les mêmes défis à relever et qu'ils font partie de la même collectivité. Ainsi, comme le soutient Sandra (2011), la société pourra assurer le bien-être de tous et d'éviter les disparités et les polarisations.

\section{References:}

1. Bazoumana, N. L. (1985). Rapport de domination et processus de différenciation catégorielle. Doctorat de 3em cycle CAEN.

2. Bazoumana, N. L. (2002). Appartenance catégorielle et formation de représentation sociales. Revue du CAMES. Série B, Vol 004.

3. Bruner, J. S. (1957). On perceptualReadiness. Psychological Review, 64, 123-152.

4. Bruner, J. S., Goodman, C. C. (1947). Value and need as organizing factors in perceptions. Journal of Abnormal and social psychology, 42, 33-44.Contributeurs

deWikipédia.(2012).Identité(sciencesociales).http://fr.wikipedia.org/ w/index.php?title=Identit\%C3\%A9_(sciences_sociales)\&oldid=8494 6028 (Page consultée le 4 novembre 2013).

5. Dacheux, E, et Goujon, d. (2010). La cohésion sociale source de la richesse économique: pour une interdisciplinaire de l'apport théorique de l'économie solidaire à la compréhension des transformations du capitalisme (communication aux XXXe journées de l'AES Charleroi, Belgique les 9 et 10 septembres 2010).

6. Deschamps, J. C. (1977). L'attribution et la catégorisation sociale. Berne, Peter Lang.

7. I.N.S. (1998). Recensement Général de la Population et de l'Habitat.

8. Durkheim, E. (1893). De la division du travail social, étude sur l'organisation des sociétés supérieures, Thèse présentée à la Faculté des lettres de Paris, Alcan, Paris, Presses Universitaires de France (réédition 1978).

9. Lewin, K. (1997). Résoudre les conflits sociaux : théorie des champs en sciences sociales. Washington D C, American Psychological Association. 
10. Mauss, M. (2007). « Essai sur le don. Forme et raison de l'échange dans les sociétés archaïques. ", L'Année sociologique. Seconde série, 1923-1924. Presses Universitaires de France, collection Quadrige.

11. Marchand, B. (1970). Auswirkw y eineremotional wertvoller Undeineremotiona lneutralem Klassifikationanf die Schätzungeiner stimulus- serie. Zeitschriftfürsozialpsychologie.

12. Mauss, M. (2007). «Essai sur le don. Forme et raison de l'échange dans les sociétés archaïques», L'année sociologique. Série 1923-1924, Presse Universitaire de France.

13. Paugam, S. (2007). Repenser la solidarité : l'apport des sciences sociales, Paris, Presses universitaires de France.

14. Picard, D. et Marc, E. (2008). Les conflits relationnels, que sais-je?, le seuil.

15. Robert, D. P. (1995). Tuning in, tuning out. The strange disappearance of social capital in America, Political Science and Politics, vol. 28, $\mathrm{n}^{\circ} 4$, p. 664-683.

16. Sandra, H. (2011). Enquête, Condition de vie et aspiration. Credoc, $\mathrm{n}^{\circ} 275$.

17. Tajfel, H. (1972). La catégorisation sociale, dans Moscovici, Introduction à la psychologie sociale. Paris, Larousse, 1 . 